\title{
Expérimenter la concertation: une clé pour des politiques environnementales plus efficaces? Une démarche multi-acteurs innovante en Tunisie centrale
}

\author{
Houssem Braiki ${ }^{1,2,3,4, *}$, Julien Burte ${ }^{1,3,4}$, Amar Imache ${ }^{5}$, Emeline Hassenforder ${ }^{4,6}$, Hamadi Habaieb ${ }^{1,7}$ \\ et Sami Bouarfa ${ }^{4,6}$ \\ ${ }^{1}$ Université de Carthage/INAT/GREEN-TEAM, 43 avenue Charles Nicolle, 1082 Tunis Mahrajène, Tunisie \\ 2 AgroParisTech, 648 rue Jean-François-Breton, 34090 Montpellier, France \\ 3 CIRAD, UMR G-EAU, 73 rue Jean-François-Breton, TA C-90/15, 34398 Montpellier, France \\ ${ }^{4}$ G-EAU, Univ Montpellier, Montpellier, France \\ ${ }^{5}$ Lisode, 2512 route de Mende, 34090 Montpellier, France \\ ${ }^{6}$ IRSTEA, UMR G-EAU, 361 rue J-F Breton, BP 5095, 34196 Montpellier cedex 05, France \\ ${ }^{7}$ Bureau de la planification et des équilibres hydrauliques, cabinet du ministre, 30 rue Alain Savary, 1002 Tunis, Tunisie
}

\begin{abstract}
Résumé - La Tunisie cherche à réorienter ses politiques d'Aménagement et de conservation des eaux et des sols (ACES) afin qu'elles gagnent en efficacité, en s'appuyant sur des démarches participatives innovantes. Cette orientation s'inscrit dans des contextes ruraux souvent peu documentés où la dimension environnementale nécessite d'être objectivée. De plus, il existe peu d'espaces de dialogue et de concertation entre les principales catégories d'acteurs des espaces ruraux concernés. Cet article analyse une démarche participative originale conçue pour produire de l'information de qualité en valorisant les savoirs locaux, partager et prendre en compte les perceptions des différents acteurs, et enfin construire des consensus pour contribuer à l'élaboration de politiques d'ACES plus efficaces. L'évaluation mobilise un double dispositif comprenant des observateurs extérieurs et une enquête de satisfaction auprès des participants. Elle s'appuie sur une grille d'évaluation de cette démarche, de ses produits et des effets induits à court terme. La structuration progressive et adaptative de la démarche, les choix des acteurs, des lieux de réalisation des ateliers et le recours à un animateur neutre ont été des facteurs très importants pour satisfaire aux critères d'évaluation de la démarche. Le partage et la prise en compte des informations et des données collectées, mais aussi des expertises et perceptions des différents acteurs, a permis de produire des informations jugées satisfaisantes ou très satisfaisantes par la totalité des participants. Cela a nourri les connaissances de la quasitotalité des acteurs et a contribué à une dynamique constructive d'apprentissage collectif. L'engagement et la mobilisation des acteurs, en particulier au niveau central, dans cet espace opérationnel de concertation sur les enjeux territoriaux, les pratiques agricoles et les ACES, ont montré une trajectoire d'ouverture vers la décentralisation du pouvoir politique.
\end{abstract}

Mots clés : approches participatives / aménagement de bassin versant / conservation des ressources naturelles / agriculture / évaluation / Tunisie

\begin{abstract}
Experiment a consultation process: a key for more efficient environmental policies? An innovating multi-stakeholders approach in central Tunisia. To render policies for water and soil conservation planning (WSCP) more efficient, Tunisia is seeking to reorient them through innovative participatory approaches. Reorientation is taking place in contexts in which data is often lacking, and where the environmental dimension needs to be clearly objectified. Furthermore, very few spaces are available that allow such dialogue between different stakeholders. This paper analyzes an original participatory approach designed to produce quality information and data by exploiting local knowledge and accounting for and sharing the perceptions of the different stakeholders, and finally building a consensus to enable more efficient WSCP policy-making. In addition to a survey of the participants' satisfaction, evaluation called on outside observers,
\end{abstract}

\footnotetext{
*Auteur de correspondance : houssem_braiki@hotmail.fr
} 
and used an evaluative framework of the process itself, its outcomes and its short-term effects. Sharing and taking into account not only the information and data collected but also the expertise and perception of the different stakeholders made it possible to produce data that all the participants rated as 'satisfactory' to 'very satisfactory'. The participatory process supported the knowledge of all the stakeholders and helped create a constructive dynamics that encouraged group learning. The commitment and the mobilization of the stakeholders, especially at national level, in an operational arena geared towards reaching agreement on territorial stakes, agricultural practices and WSCP, paves the way for the decentralization of political power.

Keywords: participatory approaches / watershed management / resource conservation / agriculture / evaluation / Tunisia

\section{Introduction}

Dans les pays du Sud, les politiques d'aménagement de l'espace rural ont évolué pour associer développement agricole et enjeu de gestion durable des ressources naturelles (Canesse, 2009). La Tunisie a une longue tradition d'investissement dans les politiques d'Aménagement et de conservation des eaux et des sols (ACES). Le bilan en est aujourd'hui questionné en termes d'appropriation sociale et d'impacts économiques et environnementaux (Chevrillon et al., 2017). Depuis les années 2000, l'État tunisien a souhaité faire évoluer ces politiques vers une démarche plus intégrée de gestion des territoires ruraux, mais les résultats obtenus sont encore en demi-teinte (Elloumi, 2006). Après la révolution de 2011, 1'enjeu d'une participation effective des populations rurales s'est renforcé, avec un objectif de passer d'une démarche d'intervention «descendante» et centralisée au niveau national, à une démarche décentralisée et horizontale incluant les acteurs des territoires (Daoud, 2011).

Les réorientations des politiques agricoles pour une prise en compte effective de la dimension environnementale nécessitent donc d'être objectivées, et ce, dans des contextes souvent peu documentés. L'un des enjeux majeurs pour les structures publiques de développement rural et la recherche tunisiennes est ainsi d'identifier, puis de renseigner, les impacts environnementaux occasionnés à la fois par les ACES et par les pratiques des agriculteurs. Un autre est clairement de faire se concerter les différentes catégories d'acteurs concernées par les politiques de développement agricole pour faire émerger des enjeux et des objectifs partagés sur lesquels pourraient s'appuyer de futures politiques d'ACES qui gagneraient ainsi en efficacité. Pour y parvenir, les démarches participatives doivent répondre à trois besoins (Romagny et Riaux, 2007) :

- produire de l'information de qualité en valorisant les savoirs locaux ;

- permettre le partage et la prise en compte des perceptions des différents acteurs;

- construire des consensus pour contribuer à l'élaboration de politiques d'ACES plus efficaces.

Dans cet article, nous présentons une démarche qui a consisté à créer un dialogue entre des acteurs administratifs du niveau central, régional et local et un groupe d'agriculteurs. Cette démarche repose sur l'hypothèse qu'une réflexion collective sur la gestion des sols et de l'eau permet de parvenir à optimiser leur usage et à les préserver (D'aquino et Seck, 2001; Mathevet et al., 2010). Elle s'inscrit alors dans une posture de recherche-action (Chia, 2004; Vall et al., 2016). Son originalité dans le contexte tunisien réside dans l'association des concepteurs classiques de ces politiques (les décideurs) et de ses «bénéficiaires » (les agriculteurs), et ce de manière ascendante.

L'objectif de cet article est d'évaluer les effets potentiels de cette démarche dans la mise en œuvre de politiques environnementales plus efficaces. De nombreuses études s'intéressent à l'évaluation des démarches participatives et de leurs impacts. Certains travaux se focalisent plus sur le déroulement de la démarche participative elle-même (Rowe et Frewer, 2000) tandis que d'autres analysent les effets, résultats ou impacts de ces démarches (Beierle 1999; Mermet et al., 2004). Il s'agit d'évaluer dans quelle mesure la démarche proposée ici confirme ou non les critères de succès avancés dans la littérature et d'évaluer sa portée à la fois sur les pratiques agricoles et sur les politiques d'ACES.

Nous présentons dans un premier temps notre démarche participative fondée sur une phase de conception et un diagnostic participatif. Nous présentons ensuite notre processus d'évaluation afin de montrer l'apport de cette démarche. Les résultats sont finalement présentés et discutés.

\section{Zone d'étude et démarche participative}

L'amont du bassin versant de Merguellil en Tunisie centrale (Fig. 1) a été choisi comme zone d'étude pour plusieurs raisons. Il a été le lieu de nombreux travaux de recherche sur le fonctionnement physique des ACES (Lacombe, 2007; Ogilvie et al., 2016) et se caractérise par un climat semi-aride (200 à $400 \mathrm{~mm} / \mathrm{an}$ de précipitations) et par des unités de production tournées vers l'élevage, l'agriculture pluviale vivrière et des cultures irriguées par les eaux souterraines. C'est dans ce contexte que les programmes d'ACES ont progressivement été implantés dans la région. Ils ont été principalement centrés sur la construction d'ouvrages, tels que les banquettes pour lutter contre l'érosion des sols et les retenues collinaires pour la recharge de la nappe (El Amami et al., 2005).

Malgré ces aménagements, les ressources en eau et en sol sont soumises à de fortes contraintes climatiques et anthropiques, leur surexploitation étant par ailleurs exacerbée par le développement de l'irrigation ou par les subventions de l'État pour les équipements d'irrigation. L'enjeu d'une meilleure efficacité des politiques publiques d'ACES est donc particulièrement important dans ces régions. Il s'agit cependant d'un contexte d'étude difficile, car:

- les questions environnementales et de durabilité à long terme sont peu considérées par les acteurs locaux;

- les connaissances socio-environnementales sur la fonction des écosystèmes sont partielles ;

- les acteurs discutent peu entre eux sur les enjeux de territoire. 


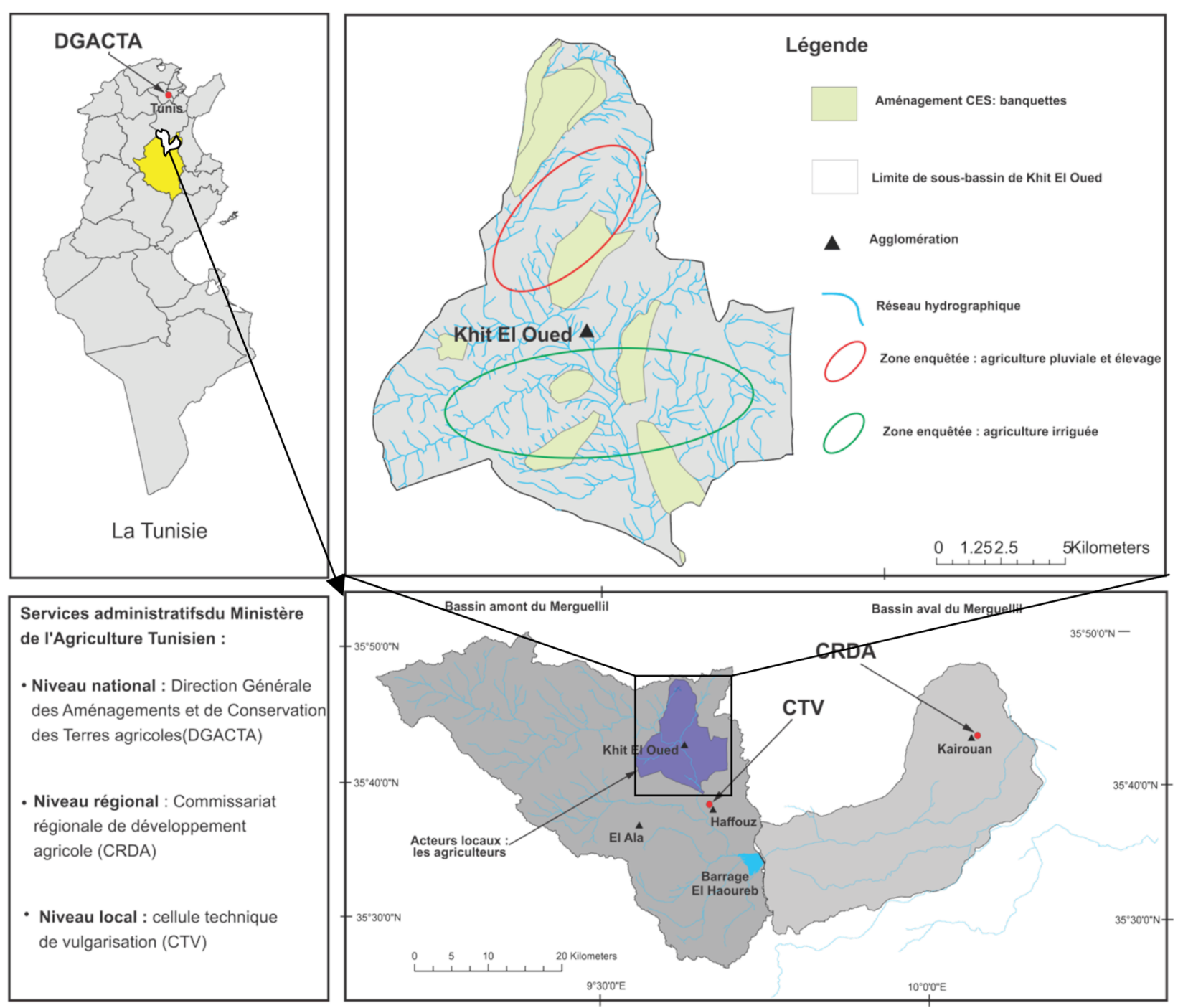

Fig. 1. Zone d'étude et acteurs mobilisés dans la démarche (acteurs locaux et services administratifs du ministère tunisien de l'Agriculture aux échelons national, régional et local).

Fig. 1. Study area and stakeholders mobilized in the process (local stakeholders and administrative services of the Tunisian Ministry of Agriculture at national, regional and local level).

Dès lors, il est essentiel d'avoir un espace effectif et constructif de dialogue qui permettrait aux acteurs du territoire de partager informations et points de vue (Glucker et al., 2013).

Notre démarche (Fig. 2) s'est structurée autour d'un diagnostic territorial participatif puis d'une succession d'ateliers par catégories d'acteurs (ou ateliers catégoriels) et d'un atelier final pluri-acteurs (Burte, 2016). Des communautés de pratiques (COMPRA) ont été élaborées avant la mise en œuvre de notre démarche. Il s'agit d'un espace de discussion qui rassemble des personnes et des chercheurs « en salle» souhaitant concevoir et tester collectivement une démarche participative en s'entraînant à faciliter les échanges entre les acteurs avant sa mise en œuvre sur le terrain (Imache et al., 2009).

Le diagnostic participatif, territorial et systémique de la zone d'étude permet de prendre contact avec les agriculteurs, de leur expliquer le sens de la démarche et de comprendre le fonctionnement du territoire au travers de leurs perceptions
(Chevrillon et al., 2017). En créant une situation d'écoute et de valorisation de leurs savoirs, il crée un climat de confiance qui permet de mobiliser les agriculteurs. Ce diagnostic a mobilisé 60 agriculteurs en entretiens individuels dans le douar de Khit El Oued. Le croisement de sources multiples d'informations (enquêtes, cartographie participative, photo-participation (encadré 1), analyse diachronique à partir d'anciens rapports) a été systématique, ce qui a permis d'accroître la précision et la qualité des données liées aux impacts des ACES.

Ce diagnostic a été suivi d'ateliers catégoriels qui avaient une double finalité. D'une part, préparer les acteurs, en particulier les agriculteurs, à l'atelier pluri-acteurs. D'autre part, ces ateliers ont permis de poursuivre la construction d'une vision partagée sur les impacts des ACES.

Les participants ont été choisis pour représenter différents points de vue et situations, mais en tenant également compte de leur capacité à échanger dans des débats multi-acteurs. Le choix des agriculteurs s'est fait en valorisant une typologie à 


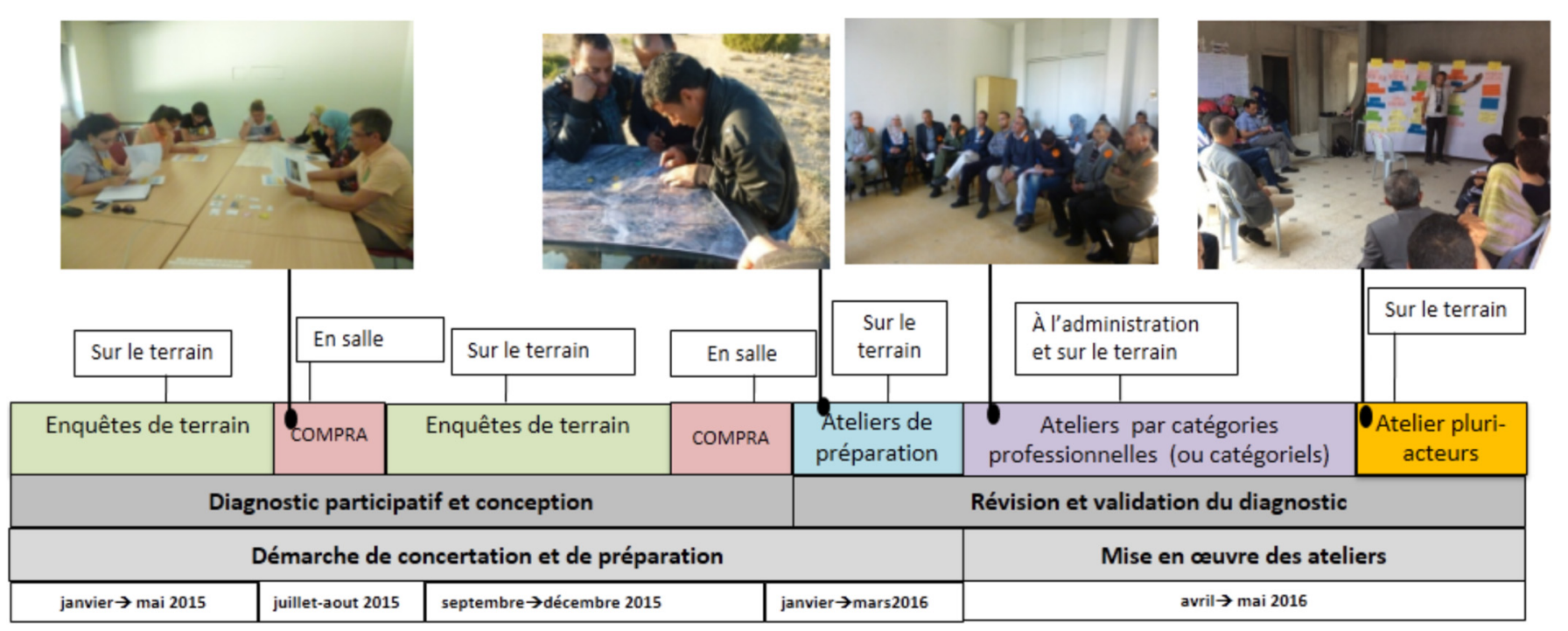

Fig. 2. Étapes de la démarche participatives sur 17 mois (COMPRA= communautés de pratique).

Fig. 2. Stages of the participatory approach over 17 months (COMPRA= communities of practice).

\section{Encadré 1. Outils de discussion.}

La cartographie participative est un espace de discussion basé sur un support cartographique. Il s'agit de faire participer les acteurs à l'identification des éléments de leur territoire, afin de les impliquer dans les actions de planification (Boutinot et al., 2008). Nous avons utilisé cet outil pour formaliser les enjeux environnementaux du territoire. L'érosion hydrique, la dégradation et la surexploitation de la nappe sont les problématiques principales du territoire qui menacent la durabilité de l'agriculture.

La photo-participation est une plateforme de discussion basée sur des photos (clichés) prises par les acteurs sur le terrain (Kankeu et Tiani, 2014), afin de mettre en débat les impacts des pratiques agricoles et les ACES. L'objectif est que les acteurs, en particulier ceux du niveau national, s'approprient le territoire.

Un jeu de rôle présente certains aspects du point de vue que les acteurs ont d'un système, en faisant jouer à ces acteurs des rôles spécifiques. Dans notre cas, il s'agissait d'identifier les impacts des ACES, mais le jeu était trop long par rapport au temps disponible; l'idée a donc été abandonnée.

dire d'acteurs préalablement élaborée comprenant quatre types (irrigants ou non, disposant d'ACES ou non). Huit agriculteurs ont été sélectionnés, soit deux par type.

Des ateliers catégoriels ont été ainsi réalisés à quatre niveaux :

- avec les agriculteurs (8 participants);

- avec les acteurs institutionnels locaux: l'administration agricole locale, la cellule technique de vulgarisation et les présidents des groupements de développement agricole (10 participants);
- avec les acteurs institutionnels régionaux : l'administration agricole régionale, le commissariat régional de développement agricole et un représentant d'une organisation non gouvernementale ( 9 participants);

- avec les acteurs institutionnels nationaux: membres de directions du ministère de l'Agriculture, d'institutions de recherche et d'enseignement supérieur ( 8 participants).

\section{Dispositif d'évaluation}

Une grille d'évaluation de notre démarche participative (Tab. 1) a été élaborée, fondée sur trois éléments: le déroulement de la démarche de concertation, les résultats obtenus et les effets induits à court terme (Rowe et Frewer, 2000, 2004 ; Mermet et al., 2004 ; Hassenforder et al., 2016).

Le cadre d'analyse proposé par Rowe et Frewer (2004) est centré sur l'efficacité d'une démarche participative à atteindre les résultats escomptés. Il invite à définir, puis opérationnaliser la définition d'efficacité la plus adaptée au terrain d'étude, puis à conduire l'évaluation de la participation et ensuite à analyser les résultats.

Comme le soulignent divers auteurs (Beierle et Cayford, 2002; Ostrom, 2005; Midgley et al., 2013; Hassenforder et al., 2016), nous sommes conscients que le contexte dans lequel se déroule la démarche participative influe sur ses résultats. Par concision, nous n'avons pas inclus de variables contextuelles dans la grille d'évaluation. Cependant, nous avons porté une attention particulière à la mise en perspective de nos résultats vis-à-vis des contraintes du territoire.

En s'inspirant du cadre d'analyse de Rowe et Frewer (2004), notre évaluation s'appuie sur différents critères (Tab. 1) et mobilise un double dispositif:

- trois observateurs extérieurs ont été attentifs à caractériser l'écoute, le ressenti et la participation par des prises de paroles volontaires lors des divers ateliers (Lapassade, 2016). Ils ont aussi noté les réactions et les comportements des participants (tensions, malentendus, conflits, accords, 
Tableau 1. Grille d'évaluation: variables, critères (1 à 10) et outils (adapté de Rowe et Frewer, 2004).

Table 1. Evaluation grid: variables, criteria (1 to 10) and tools (adapted from Rowe et Frewer, 2004).

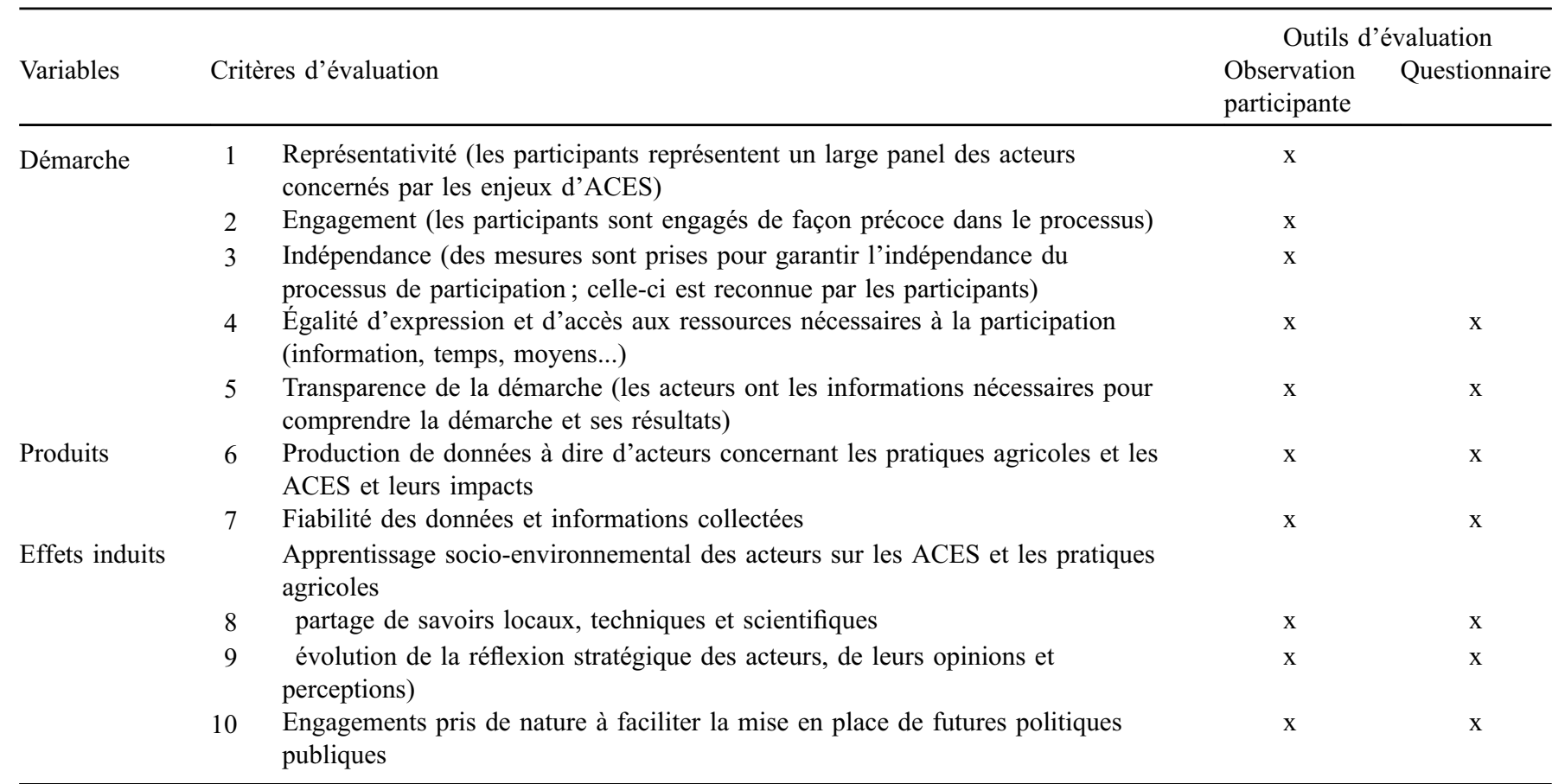

désaccords, rapports hiérarchiques) et leurs répercussions sur la démarche;

- un questionnaire d'évaluation distribué à la fin de la démarche a permis à chaque participant d'exprimer son point de vue, sa satisfaction ou son mécontentement sur le déroulé des ateliers et ses résultats et effets induits. Cette évaluation s'appuie sur une échelle qualitative à quatre niveaux d'atteinte de l'objectif (très insatisfaisant, insatisfaisant, satisfaisant, très satisfaisant).

\section{Résultats}

\section{1 Évaluer le déroulement de la démarche: participation et niveau de satisfaction des acteurs}

\section{Q2 4.1.1 La représentativité}

Les différents niveaux de décision de l'administration et les différents domaines de compétence concernés par ce diagnostic sont représentés. Les institutions de recherche tunisiennes étaient présentes (Institut national de recherches en génie rural, eaux et forêts [INRGREF] et Institut national agronomique de Tunisie [INAT]). Les agriculteurs présents durant les ateliers représentent les quatre types correspondant à la typologie établie du territoire.

\subsubsection{L'engagement}

Les agriculteurs étaient fortement intéressés par l'opportunité, inédite pour eux, d'échanger avec des hauts responsables de l'administration pendant l'atelier mixte. Ils ont cependant souligné les limites dans l'obtention de réponses à leurs questions et d'avis relatifs à leurs propositions concrètes qui dépassaient largement le cadre de l'ACES. Pour l'administration, il s'agissait d'une opportunité de partager l'expertise entre des techniciens locaux et leurs chefs au niveau central, ainsi que les points de vue des représentants des agriculteurs. L'engagement des acteurs dans les ateliers a été facilité par l'usage d'outils innovants tels que la cartographie participative et la photo-participation (encadré 1): «on a hâte de savoir quels seront les résultats que l'on obtiendra avec ces nouveaux outils».

\subsubsection{La neutralité}

L'animation a été réalisée par le premier auteur de l'article qui a été formé avec un bureau d'étude spécialiste en ingénierie de concertation. Il connaissait les différentes parties prenantes (administrations et agriculteurs), avec lesquelles il avait des liens de confiance. Étant chercheur, il n'était pas une partie prenante des impacts des pratiques agricoles et des ACES. Sa position de neutralité dans le débat était ainsi garantie. Il a facilité le dialogue et il a veillé à ce que les agriculteurs contribuent équitablement à la discussion.

\subsubsection{L'égalité d'expression et d'accès aux ressources nécessaires à la participation (Fig. 3)}

Tous les acteurs ont d'abord pu s'exprimer dans les ateliers catégoriels réalisés «chez eux». L'atelier multi-acteurs a été réalisé sur le territoire d'étude, ce qui a renforcé la confiance des agriculteurs à prendre librement la parole. Cela a contribué à réduire les asymétries de pouvoir. Les discussions relatives aux effets et impacts des ACES (par exemple, augmenter la production $v s$. réduire la surface agricole utile) ont été dominées surtout par les agriculteurs. L'animateur, attentif à 


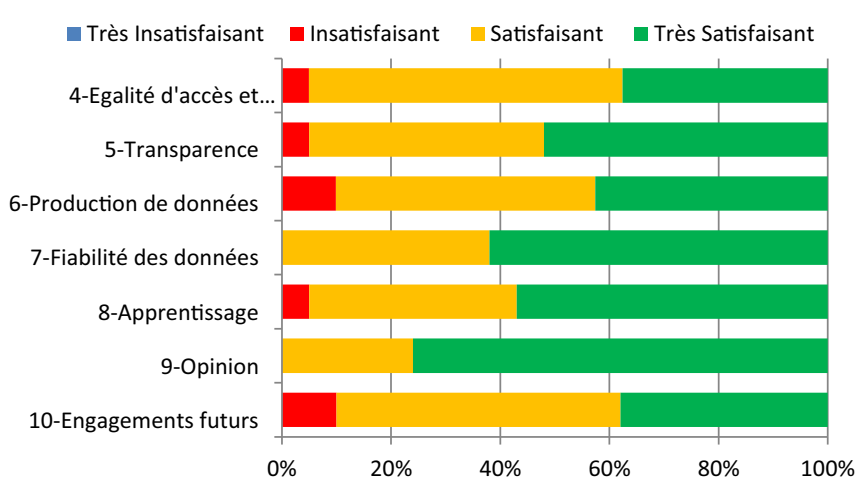

Fig. 3. Résultats du questionnaire (très insatisfaisant, insatisfaisant, satisfaisant, très satisfaisant).

Fig. 3. Results of the questionnaire (very unsatisfactory, unsatisfactory, satisfactory, very satisfactory).

l'expression de chacun et à ne pas reproduire de hiérarchie administrative ou d'asymétrie de pouvoir, a modéré les débats dans ce sens. Globalement, $95 \%$ des participants ont été satisfaits sur l'égalité de temps de parole.

\subsubsection{La transparence (Fig. 3)}

Tous les acteurs ont été informés de chaque étape de la démarche, ses objectifs et son déroulement. Les résultats de chaque étape de la démarche participative ont été validés par les différentes catégories d'acteurs. Parmi les participants, $95 \%$ ont exprimé une opinion positive sur ce point.

L'évaluation a posteriori met en évidence trois points cruciaux pour la réussite de la démarche en termes de participation effective :

- le choix des acteurs détermine leur capacité et leur légitimité à représenter leur territoire et leurs pairs. L'implication des acteurs en amont de la démarche a contribué à sa réussite ;

- la structuration progressive et adaptative de la démarche a permis de préparer les acteurs à l'exercice de concertation pluri-acteurs de l'atelier mixte, et ainsi de limiter les asymétries de pouvoir et de connaissance (connaissances techniques, connaissance du territoire, etc.);

- le choix des lieux des ateliers a contribué à la facilité d'expression et de participation des acteurs.

\section{2 Évaluer la robustesse des produits}

Les produits bruts les plus importants ont été :

- le diagnostic territorial systémique basé sur des cartes, des tableaux d'impacts et des indicateurs;

- la caractérisation des pratiques productives et d'ACES ;

- l'évaluation qualitative de leurs impacts en s'attachant à comprendre quels indicateurs étaient mobilisés par chaque acteur.

La totalité des acteurs a évalué la fiabilité des données et informations comme satisfaisantes ou très satisfaisantes (Fig. 3). La triangulation, les débats et discussions itératifs ont contribué à renforcer leur robustesse, leur fiabilité et leur légitimité. Cela est montré par les nombreuses modifications ou compléments apportés au diagnostic et évaluation par les participants au cours du processus.

\section{3 Évaluer les effets induits à court terme de la démarche en terme d'apprentissage collectif}

La démarche, qui s'est étalée sur un an et demi, a contribué à l'évolution des perceptions et à une plus forte compréhension mutuelle des agents de l'administration, en particulier nationaux, et des agriculteurs. L'opinion initiale des acteurs nationaux était que les agriculteurs ne comprenaient pas l'intérêt des ACES car ils manquaient de connaissances sur leurs impacts. Ils proposaient, en ce sens, l'organisation de journées de vulgarisation et sensibilisation, et donc la reproduction d'un schéma vertical top-down. Parallèlement, les agriculteurs considéraient que l'entretien des ACES était de la responsabilité de l'administration. Plus globalement, les agriculteurs se sentaient abandonnés par l'administration, surtout après la révolution de 2011. Les échanges lors des ateliers ont fait évoluer ces perceptions et ils ont finalement abouti à ce que l'ensemble des acteurs propose et s'accorde sur l'intérêt de la création d'un espace institutionnel opérationnel et multi-acteurs d'échanges autour des enjeux du territoire.

La démarche a contribué à un apprentissage collectif sur le plan socio-environnemental.

Les agriculteurs affirment avoir appris sur l'entretien des banquettes ainsi que sur leur utilité. De plus, ils ont mieux cerné les quantités d'engrais chimiques à apporter afin d'améliorer la fertilité de leurs sols selon le type de culture. L'administration affirme avoir amélioré ses connaissances sur les impacts des banquettes installées près des oliviers, et apprécie en particulier d'avoir des données précises sur leurs rendements (le rendement atteint avec banquettes est trois fois supérieur: $140 \mathrm{~kg}$ au lieu de $45 \mathrm{~kg}$ ).

Le partage et la prise en compte des informations et des données collectées, mais aussi des expertises et perceptions des différents acteurs, ont nourri les connaissances de l'ensemble des acteurs et ont contribué à une dynamique constructive d'apprentissage collectif. Les agriculteurs ont ainsi reconnu à la fin de la démarche que: "Malgré le fait qu'[ils soient] des agriculteurs de père en fils, [leurs] connaissances sur le rôle et les impacts des ACES se sont améliorées après avoir discuté avec les différents acteurs présents».

\section{Discussion et conclusion}

\subsection{L'apport de la démarche: vers une dynamique de développement territorial}

Clairement, l'engagement des acteurs dans cette démarche a été facilité par un contexte tunisien post-révolutionnaire. Les bailleurs de fonds qui financent les projets de développement agricole et d'aménagements en Tunisie mobilisent des bureaux d'ingénieurs-conseil pour faire le travail de diagnostic (absence de fiabilité des données). Les approches participatives dans ces projets ont été marquées par une voie descendante et un faible espace accordé à la population locale (Elloumi, 2006). Elles ont essentiellement contribué à trouver des solutions techniques telles que la réparation des vannes 
dans un GDA (Al Atiri, 2006). L'approche mise en œuvre ici est plus «ascendante» et part des perceptions de terrain (les agriculteurs) et d'un choix de construction des stratégies au niveau local. Notre démarche est participative et multi-acteurs en même temps. Enfin, elle innove dans le sens où les acteurs sont tous impliqués dans la réflexion sur les indicateurs et la co-construction d'une grille commune, permettant une évaluation partagée des impacts des ACES et des pratiques agricoles, démarche inédite à notre connaissance.

Les agriculteurs ont proposé de créer une association: «on pourra discuter des problèmes du territoire et réfléchir ensemble à des solutions. En plus, on pourra plus facilement garder le contact avec l'administration». Après l'atelier multi-acteurs, les décideurs au niveau national ont également fait part de l'idée de constituer un comité de territoire pour assurer la durabilité du dialogue et la concertation entre les acteurs. Les agriculteurs: "On veut un comité [de territoire] formé des agriculteurs et des agents de l'administration dans le but de nous organiser et de nous créer un [espace de] dialogue pour mieux résoudre nos problèmes ». Les échanges au cours de l'atelier ont conforté les décideurs nationaux dans leur idée de s'appuyer sur de tels comités pour leur prochain projet d'ACES et développement rural (projet PACTE). Dans un contexte comme celui de la Tunisie actuellement, ce type de démarche multi-acteurs semble donc prometteur pour nourrir de nouvelles dynamiques de développement territorial concertées. Reste à savoir si ces souhaits, en l'absence d'appui apporté par la recherche, se verront suivis d'effet ou non.

\subsection{Les limites de la démarche}

Plusieurs limites doivent être soulignées. Une des principales contraintes est l'investissement conséquent nécessaire en temps, pour la construction comme pour la mise en œuvre. Ainsi, lors des Communautés de pratique, le test d'un jeu de rôle a montré que le développement du jeu serait trop long par rapport au temps disponible : l'idée de s'appuyer sur un jeu a alors été abandonnée.

Des tensions entre des acteurs étaient prévisibles. Ces tensions se sont effectivement matérialisées dans l'atelier multi-acteurs du côté de l'administration avec des remarques typiques: "les agriculteurs doivent comprendre qu'ils doivent protéger les ressources en eau et sol» ou de la part des agriculteurs : «l'administration nous a abandonnés, quand on a besoin d'un conseil technique, personne ne vient $\gg$ ou encore «à quoi sert l'administration?». Éviter totalement ces expressions, fruits d'une longue histoire de défiance, est bien entendu impossible et nous nous sommes employés à en limiter la portée pour orienter le plus possible le débat sur la question des ACES. Cela a été fait:

- en sensibilisant en amont les différents acteurs au dialogue et à l'écoute ;

- en tenant compte de ce facteur dans l'architecture de l'atelier multi-acteurs, via la programmation d'une séquence consacrée à un temps de «libre expression et d'écoute», qui a pu être utilisé par certains participants pour faire passer un message qui leur tenait à cœur.

\subsection{La portée de la démarche: une clé pour des politiques publiques plus efficaces}

Des enjeux partagés, tels que l'érosion des terres et la surexploitation de la nappe, sur lesquels pourraient s'appuyer de futures politiques d'ACES plus pertinentes, ont émergé. Notre démarche a permis le partage et la prise en compte des perceptions des différents acteurs et construit des consensus propices à aider à l'élaboration de politiques d'ACES plus efficaces.

Cependant, les acteurs du territoire n'ont participé ni à l'identification des objectifs ni à l'analyse des données qui ont été faites par notre équipe de recherche. Mais la démarche demeure participative dans la mesure où les acteurs ont occupé un espace de dialogue et de concertation opérationnel, jusquelà inexistant sur leur territoire et qui a débouché sur des produits concertés prometteurs.

L'architecture de notre démarche a créé une forte capacité d'innovation procédurale et a révélé chez les parties prenantes des caractères d'adaptabilité, de progressivité et d'itérativité qui ont contribué à sa réussite. Réunir les acteurs du développement et de la recherche pour la conception et la conduite de telles démarches participatives, en intégrant le plus amont possible les acteurs du territoire, nous semble être une clé de succès prometteuse et profitable à tous. Il convient désormais de mener d'autres recherches innovantes et opérationnelles dans ce sens afin de renforcer davantage la collaboration autonome et durable entre les parties prenantes, tout en respectant la place qui revient à chacun.

Remerciements. Nous tenons à remercier l'UMR G-eau du Cirad (www.geau.net), le réseau SIRMA (www.rcp-sirma. org), les coordonnateurs des projets de recherche ANR GroundwaterArena et Amethyst, et des projets de recherchedéveloppement AFD/DGACTA et PROSCAR, ainsi que nos partenaires de la DGACTA-MARHP.

\section{Références}

Al Atiri R. 2006. Évolution institutionnelle et réglementaire de la gestion de l'eau en Tunisie. Vers une participation accrue des usagers de l'eau. In: Actes du colloque L'avenir de l'agriculture irriguée en Méditerranée. Nouveaux arrangements institutionnels pour une gestion de la demande en eau, Cahors, France. https://hal. archives-ouvertes.fr/cirad-00191075/document.

Beierle TC. 1999. Using social goals to evaluate public participation in environmental decisions. Review of Policy Research 16(3-4): 75-103. DOI: 10.1111/j.1541-1338.1999.tb00879.x.

Beierle TC, Cayford J. 2002. Democracy in practice: public participation in environmental decisions. First ed. Resources for the Future. Washington D.C. http://www.rff.org/files/sharepoint/ WorkImages/Download/RFF-DP-99-06.pdf.

Boutinot L, Viau AA, Leclerc G. 2008. Questions sur la neutralité des outils de type jeux de rôle et cartographie participative dans une expérience de gouvernance foncière au Sénégal. Norois Environnement, Aménagement, Société 209: 73-89. DOI: 10.4000/ norois.2641.

Burte J. 2016. Diagnostic rapide participatif systémique: guide pratique. Tunis : CIRAD-ES, UMR G-EAU, 19 p. http://agritrop. cirad.fr/584066/. 
Canesse AA. 2009. Gestion des ressources naturelles et système institutionnel de gouvernance en Tunisie. Maghreb-Machrek 4 (202): 49-64. DOI: 10.3917/machr.202.0049.

Chevrillon A, Ben Haha N, Burte J. 2017. Vers une territorialisation des politiques rurales en Tunisie: l'exemple des politiques de conservation des eaux et des sols. In : Caron P, Valette E, Wassenaar T, Coppens d'Eeckenbrugge G, Papazian V, eds. Des territoires vivants pour transformer le monde. Versailles : Quae, pp. 172-178. http://agritrop.cirad.fr/583571/.

Chia E. 2004. Principes, méthodes de la recherche en partenariat : une proposition pour la traction animale. Revue d'Élevage et de Médecine Vétérinaire des Pays Tropicaux 57(3-4): 233-240. DOI: 10.19182/remvt.9895.

Daoud A. 2011. La révolution tunisienne de janvier 2011 : une lecture par les déséquilibres du territoire. EchoGéo. DOI: 10.4000/echogeo.12612.

D'aquino P, Seck SM. 2001. Et si les approches participatives étaient inadaptées à la gestion décentralisée de territoire ? Géo-carrefour 76: 233-239. DOI: 10.3406/geoca.2001.2561.

El Amami H, Bachta MS, Nasri S, Cudennec C. 2005. Allocation des ressources en eaux sous des contraintes économiques, sociales et environnementales. Cas du bassin de Merguellil en Tunisie centrale. In: Actes du colloque Les instruments économiques et la modernisation des périmètres irrigués. Kairouan (Tunisie): Cirad, 14 p. http://hal.cirad.fr/cirad-00193646/document.

Elloumi M. 2006. Les politiques de développement rural en Tunisie : acquis et perspectives. Montpellier: CIHEAM, Options Méditerranéennes : Série A, Séminaires Méditerranéens, $\mathrm{n}^{\mathrm{o}} 71$, pp. 55-65. http://om.ciheam.org/om/pdf/a71/06400057.pdf.

Glucker A, Driessen N, Kolhoff P, Runhaar HA. 2013. Public participation in environmental impact assessment: why, who and how? Environmental Impact Assessment Review 43: 104-111. DOI: 10.1016/j.eiar.2013.06.003.

Hassenforder E, Pittock J, Barreteau O, Daniell, KA, Ferrand N. 2016. The MEPPP framework: a framework for monitoring and evaluating participatory planning processes. Environmental Management 57(1): 79-96. DOI:10.1007/s00267-015-0599-5.

Imache A, Dionnet M, Bouarfa S, Jamin JY, Hartani T, Kuper M, et al. 2009. "Scénariologie participative»: une démarche d'apprentissage social pour appréhender l'avenir de l'agriculture irriguée dans la Mitidja (Algérie). Cahiers Agricultures 18(5): 417-424. DOI: 10.1684/agr.2009.0324.

Kankeu RS, Tiani AM. 2014. Guide de cartographie participative géoréférencée pour la gestion communautaire du terroir. Indonésie : CIFOR, vol. 158. http://www.cifor.org/publications/pdf_files/WPa pers/WP158Kankeu.pdf.
Lacombe G. 2007. Évolution et usage de la ressource en eau dans un bassin versant aménagé semi-aride : le cas de Merguellil en Tunisie centrale. Thèse de doctorat en Eaux Continentales et Société. Univ. Montpellier 2, 304 p. http://horizon.documentation.ird.fr/exl-doc/ pleins_textes/divers16-05/010043055.pdf.

Lapassade G. 2016. Observation participante. In: Barus-Michel J, Enriquez E, Lévy A, eds. Vocabulaire de psychosociologie. Références et positions. Toulouse: ERES, pp. 392-407. DOI: 10.3917/eres.barus.2016.01.0392.

Mathevet R, Thompson J, Delanoë O, Cheylan M, Gil-Fourrier C, Bonnin $\mathrm{M}$, et al. 2010. La solidarité écologique: un nouveau concept pour une gestion intégrée des parcs nationaux et des territoires. Natures Sciences Sociétés 18(4): 424-433. DOI: $10.1051 / \mathrm{nss} / 2011006$.

Mermet L, Dubien I, Emerit A, Laurans Y. 2004. Les porteurs de projets face à leurs opposants : six critères pour évaluer la concertation en aménagement. Politiques et Management Public 22 (1): 1-22. DOI: 10.3406/pomap.2004.2829.

Midgley G, Cavana RY, Brocklesby J, Foote JL, Wood DRR, AhuririDriscoll A. 2013. Towards a new framework for evaluating systemic problem structuring methods. Eur J Oper Res 229(1): 143e154. DOI: 10.1016/j.ejor.2013.01.047.

Ogilvie A, Le Goulven P, Leduc C, Calvez R, Mulligan M. 2016. Réponse hydrologique d'un bassin semi-aride aux événements pluviométriques et aménagements de versant (bassin du Merguellil, Tunisie centrale). Hydrological Sciences Journal 61(2): 441-453. DOI:10.1080/02626667.2014.9344249.

Ostrom E. 2005. Understanding Institutional Diversity. Princeton: Princeton University Press. http://press.princeton.edu/chapters/ s8085.pdf.

Romagny B, Riaux J. 2007. Community-based agricultural water management in the light of participative policies: a cross-cultural look at cases in Tunisia and Morocco. Hydrological Sciences Journal/Journal des Sciences Hydrologiques 52(6): 1179-1196. DOI: $10.1623 /$ hysj.52.6.1179.

Rowe G, Frewer LJ. 2000. Public participation methods: a framework for evaluation. Science, Technology \& Human Values 25(3): 3-29. DOI:10.1177/016224390002500101.

Rowe G, Frewer, LJ. 2004. Evaluating public participation exercices. A research agenda. Science, Technology \& Human Values 29(4): 512-556. DOI:10.1177/0162243903259197.

Vall E, Chia E, Blanchard M, Koutou M, Coulibaly K, Andrieu N. 2016. La co-conception en partenariat de systèmes agricoles innovants. Cahiers Agricultures 25(1): 15001. DOI: 10.1051/cagri/ 2016001.

Citation de l'article : Braiki H, Burte J, Imache A, Hassenforder E, Habaieb H, Bouarfa S. 2018. Expérimenter la concertation : une clé pour des politiques environnementales plus efficaces? Une démarche multi-acteurs innovante en Tunisie centrale. Cah. Agric. 27 : 15003. 